\title{
Cellular Internalization of Therapeutic Oligonucleotides by Peptide Amphiphile Nanofibers and Nanospheres
}

\author{
Didem Mumcuoglu, ${ }^{\dagger, \#}$ Melis Sardan Ekiz, ${ }^{\dagger, \#}$ Gokhan Gunay, ${ }^{\dagger}$ Turgay Tekinay, ${ }^{\ddagger}$, Ayse B. Tekinay, ${ }^{*}{ }^{\dagger}$ \\ and Mustafa O. Guler* ${ }^{\dagger}$
}

\begin{abstract}
${ }^{\dagger}$ Institute of Materials Science and Nanotechnology, National Nanotechnology Research Center (UNAM), Bilkent University, Ankara 06800, Turkey

${ }^{\ddagger}$ Life Sciences Application and Research Center and ${ }^{\S}$ Department of Medical Biology and Genetics, Faculty of Medicine, Gazi University, Ankara 06500, Turkey
\end{abstract}

Supporting Information

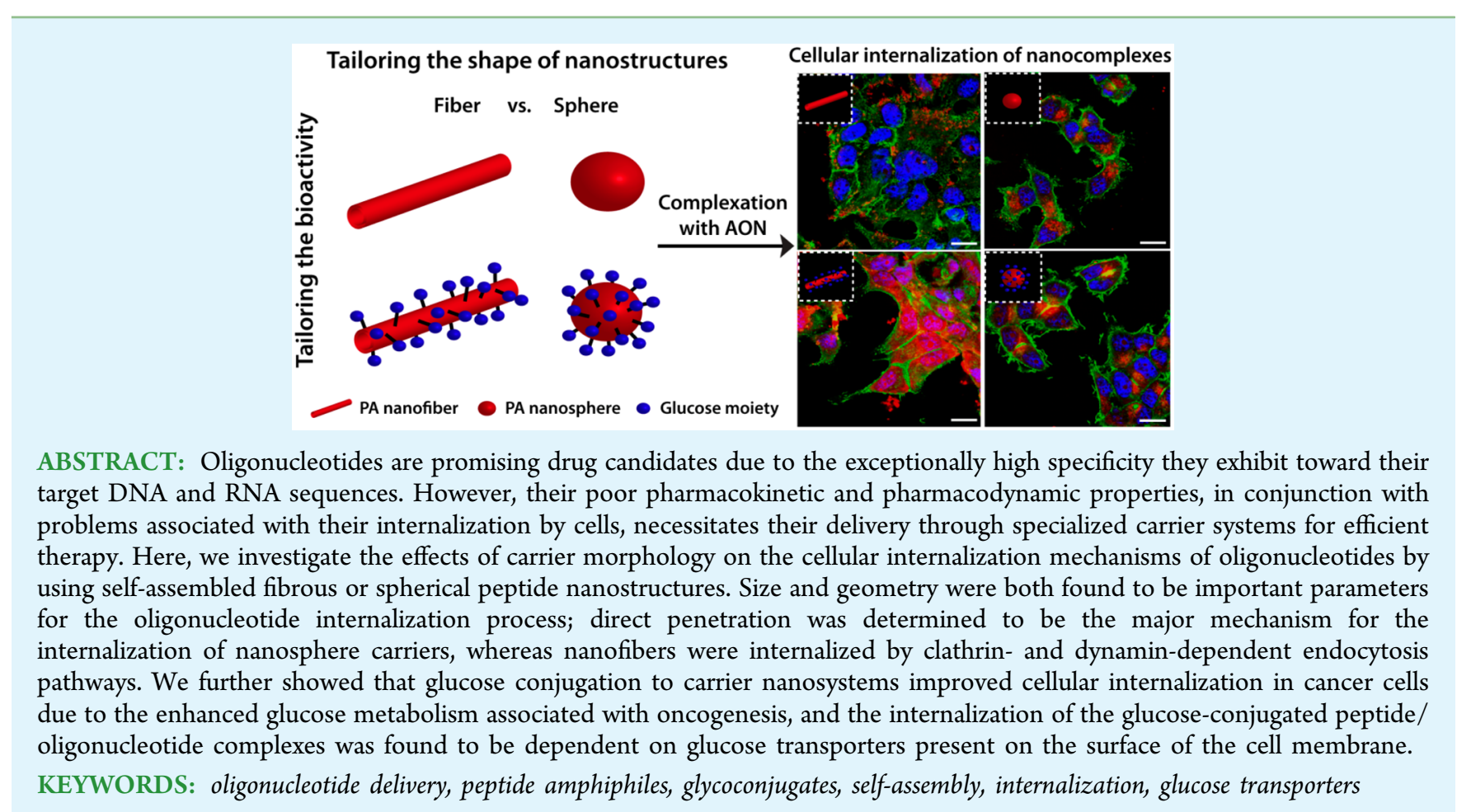

\section{INTRODUCTION}

Therapeutic oligonucleotides such as aptamers, CpG oligonucleotides, siRNAs, and antisense oligonucleotides have received considerable attention in the pharmaceutical industry for their ability to selectively inhibit the activity of nucleic acid sequences. $^{1-3}$ Unlike small molecule drugs, oligonucleotides can be designed to specifically target a broad range of biologically relevant mechanisms, which makes them highly attractive molecules for the treatment of conditions such as cardiovascular diseases, metabolic diseases, cancer, and genetic disorders. $^{3-5}$ However, oligonucleotide drugs invariably suffer from high degradation rates and short half-lives in the plasma, and although their stability can be improved through chemical modification, their susceptibility to nucleolytic enzymes and rapid clearance by renal filtration and reticuloendothelial activity are nonetheless major issues for the success of their pharmaceutical applications. Consequently, many oligonucleo- tide-based clinical trials have suffered due to problems associated with low biodistribution and poor pharmacokinetic and pharmacodynamic properties. 6,7

Drug delivery systems are able to prevent the exposure of drug molecules to biological environments, greatly increasing their stability and improving their pharmacokinetic and pharmacodynamic properties. As such, delivery vehicles are an ideal means of bypassing the limitations of oligonucleotidebased therapeutic agents. Carrier systems intended for oligonucleotide delivery should be able to protect their cargo from enzymatic degradation and immune system recognition and prevent their reticuloendothelial system clearance and rapid renal excretion. The cellular internalization of oligonu-

Received: February 4, 2016

Accepted: April 20, 2016

Published: April 20, 2016 
Scheme 1. Schematic Representations of Peptide Amphiphiles and Their Self-Assembly into Nanospheres or Nanofibers

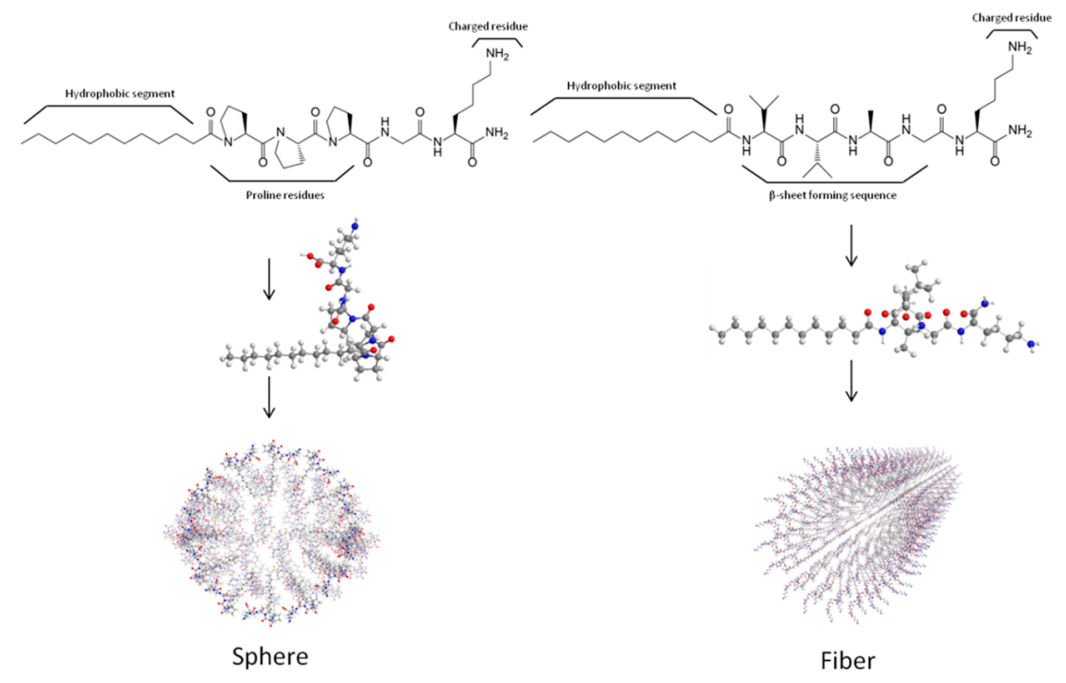

a)

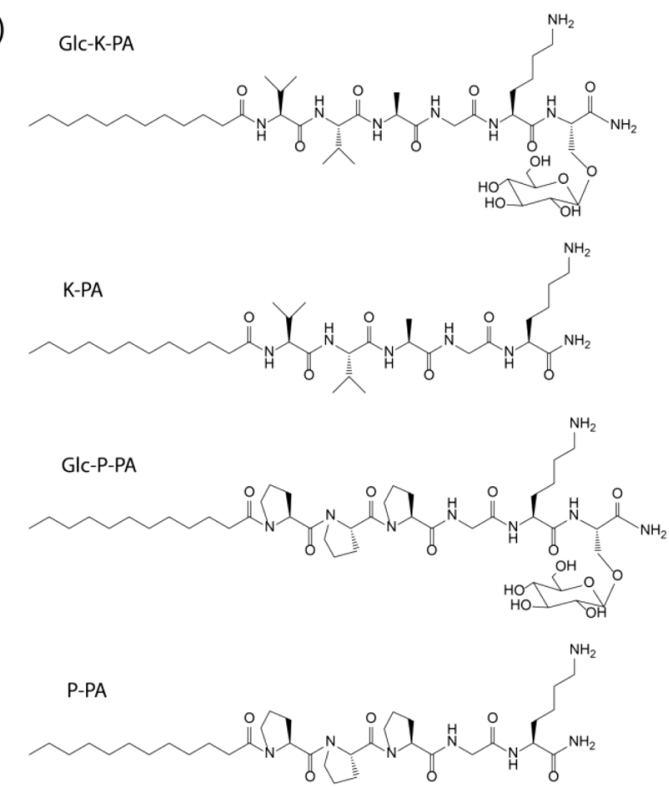

b)
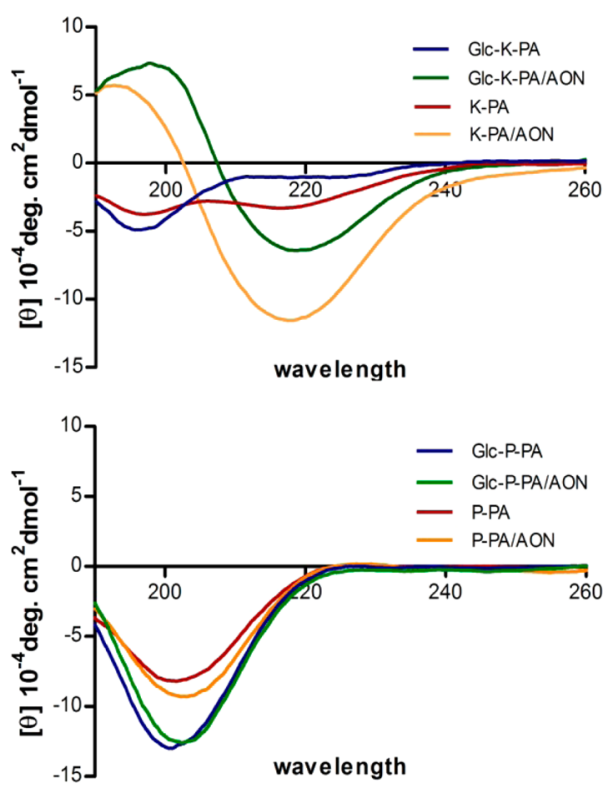

Figure 1. (a) Chemical structures of amphiphilic glycopeptides Lauryl-VVAGKS( $\beta$-D-Glc)-Am (Glc-K-PA) and Lauryl-P ${ }_{3}$ GKS $(\beta$-D-Glc)-Am $($ GlcP-PA) and peptide amphiphiles Lauryl-VVAGK-Am (K-PA) and Lauryl-P ${ }_{3}$ GK-Am (P-PA). (b) CD spectra of PA and PA/AON complexes.

cleotides has been shown to be increased when cationic lipids, ${ }^{8}$ polymers, ${ }^{9}$ and nanoparticles ${ }^{10-12}$ are used as carrier systems. However, the size and morphology of a carrier system are also important for determining the mechanisms of its internalization; thus, a better understanding of these factors and their impacts is vital for the development of new delivery systems. Nevertheless, the effect of size and geometry in drug delivery has previously been described only for a limited number of materials and cell types, and further investigation is necessary to improve our basic understanding of the carrier internalization process. $^{13,14}$

Glucose molecules can be utilized for targeting tumor tissue, as cancer cells tend to overexpress glucose receptors to meet their heightened metabolic demands. ${ }^{15}$ This tendency, which typically accompanies a switch from oxidative phosphorylation to glycolysis for ATP production, ${ }^{16}$ is known as the Warburg effect $^{17}$ and is considered to be one of the hallmarks of cancer. ${ }^{18}$ Consequently, cancer cells consume more glucose than their healthy counterparts and must overexpress certain glucose transporters to increase their glucose uptake. The glucose transporter GLUT1, for example, is overexpressed in many cancer types, including breast cancer, ${ }^{19}$ renal cell carcinomas (RCCs), ${ }^{20}$ and colorectal cancer. ${ }^{21}$ Glucose transporters can also be targeted to monitor cancer activity and progression, as the expression of GLUT1 was found to be correlated with invasiveness of breast cancer ${ }^{22}$ and inversely correlated with prognosis of cancer. ${ }^{23}$ Other than the GLUT family, the expression of sodium-dependent glucose transporters (SGLT1 and SGLT2) was also found to be related to cancer survival in pancreatic adenacarcinoma ${ }^{24}$ and metastasis of lung cancer. ${ }^{25}$ Glucose-conjugated anticancer drugs have been shown to target tumor tissue based on increased glucose uptake in cancer cells: glufosfamide, ${ }^{26}$ paclitaxel, ${ }^{27}$ doxorubicin, $^{28}$ and tamoxifen ${ }^{29}$ glycoconjugates were synthesized and tested for cancer targeting, and glucose conjugation was found to enhance the solubility and cellular uptake of these drugs. Many clinical trials also utilized glycoconjugates for targeting tumors and decreasing side effects of chemotherapeutics. ${ }^{30}$ The 
effective dose in glycoconjugates can be decreased compared to that in nonconjugated anticancer drugs; however, a major drawback of glycoconjugate drugs is that the drug chemical structure changes after this conjugation and glucose needs to be enzymatically cleaved off for pharmaceutical activity. ${ }^{31}$ Noncovalent functionalization can eliminate this drawback, and glucose-functionalized polymeric micelles were recently described for this purpose. 32,33

Self-assembled peptide amphiphile (PA) nanofibers have previously been shown to be effective for antisense oligonucleotide (AON) delivery. ${ }^{34} \mathrm{PA}$ molecules are biocompatible and their degradation products are natural biomolecules that can be metabolized in the body, which allows their use as delivery agents with minimal side effects. Peptide-based carrier nanostructures are also easy to produce and self-assemble directly following the mixing of oligonucleotides with PAs. The sequences of the peptides can also be designed to incorporate cancer-targeting moieties for specific delivery into tumors. In this work, we investigate the effect of morphology on the delivery efficiency of two distinct PA-based carrier systems, which were designed to self-assemble into nanofibers or nanospheres by tuning the noncovalent interactions responsible for triggering the aggregation of the PA molecules (Scheme 1). In addition to carrier morphology, we also utilized glucose functionalization to study the mechanisms involved in the internalization of glucose-functionalized and nonfunctionalized delivery vehicles.

\section{RESULTS AND DISCUSSION}

Peptide amphiphile molecules (K-PA and P-PA) and amphiphilic glycopeptides (Glc-K-PA and Glc-P-PA) in Figure 1 were synthesized using solid phase peptide synthesis and characterized by LC-MS (Figure S1). Glc-K-PA and K-PA were designed with a Val-Val-Ala peptide sequence followed by a hydrophilic lysine residue to allow their complexation with oligonucleotides, and this motif was replaced with three proline residues in Glc-P-PA and P-PA. The targeting molecule Dglucose $(\beta$-D-Glc) was conjugated onto the peptide sequence by using serine-linked glucose for both Glc-K-PA and Glc-P$\mathrm{PA}$. Whereas the Val-Val-Ala sequence facilitates $\beta$-sheet formation through hydrogen bonding among the peptide molecules, proline residues serve as $\beta$-sheet breakers by disrupting $\beta$-sheet secondary interactions. ${ }^{35,36}$

The self-assembly mechanisms of these systems are shown in Scheme 1. When dissolved in an aqueous solution, PA molecules self-assemble into nanostructures that contain hydrophobic amino acid residues and an alkyl tail on their interior and present their hydrophilic sections on their exterior. Glc-K-PA and K-PA molecules, which contain $\beta$-sheet forming regions and exhibit a positive charge at neutral $\mathrm{pH}$, form cylindrical nanostructures as a result of their complexation with negatively charged oligonucleotides. In contrast, Glc-P-PA and P-PA possess $\beta$-sheet breaking proline residues and form spherical nanostructures upon the neutralization of their lysine side chain with oppositely charged oligonucleotides. In addition, both of the amphiphilic glycopeptides (Glc-P-PA and Glc-K-PA) self-assembled into nanostructures that present the glucose unit on their periphery, further enhancing cellmaterial interactions.

The secondary structure (Figure $1 \mathrm{~b}$ ), morphology (Figure 2), size (Figure S2), and zeta potentials (Table S1) of the selfassembled peptide-oligonucleotide nanostructures were studied. The secondary structures of the PAs were investigated with

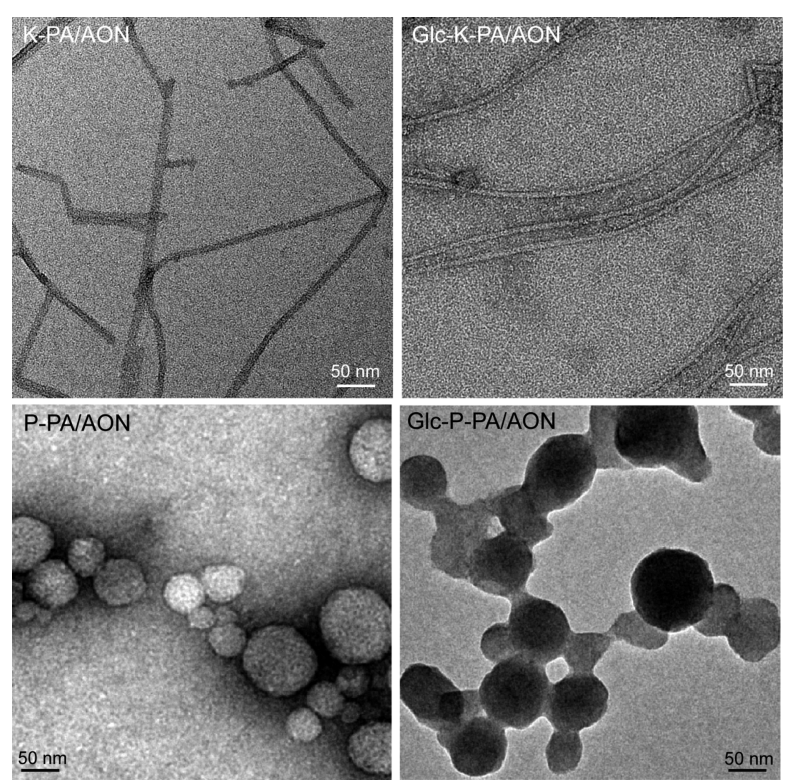

Figure 2. TEM images of PA/AON complexes at a 30:1 PA/AON molar ratio. Scale bars: $50 \mathrm{~nm}$.

circular dichroism (Figure 1b), and Glc-K-PA and K-PA were found to form $\beta$-sheet structures in the presence of AONs at physiological $\mathrm{pH}$, as suggested by a positive peak at around 193 $\mathrm{nm}$ and a negative peak at around $217 \mathrm{~nm}$. In the absence of AONs, the negative peak at 217 was weakened in the K-PA solution and disappeared entirely in the Glc-K-PA solution, and a negative peak at $197 \mathrm{~nm}$ appeared for both PAs, indicating a random coil conformation. On the other hand, the proline-rich PAs Glc-P-PA and P-PA showed mixtures of random coil and poly proline helix (PPII) secondary structures with a strong negative band at $203 \mathrm{~nm}$ and weak positive band at 226 nm. ${ }^{37-39}$ The secondary structures of proline-rich peptides were preserved after their complexation with AONs.

TEM images were acquired to observe the morphologies of nanostructures in the presence of AONs (Figure 2). K-PA and Glc-K-PA molecules formed nanofibers in the presence of AONs with fiber diameters of approximately $10 \mathrm{~nm}$. The presence of AONs appears to allow a complete self-assembly process with $\beta$-sheets as the predominant secondary structure, supporting our CD results. TEM images of P-PA/AON and Glc-P-PA/AON complexes, in contrast, suggest that the proline-rich PAs assemble into spherical nanostructures with diameters of $50-100 \mathrm{~nm}$. The hydrodynamic sizes of P-PA/ AON and Glc-P-PA/AON nanospheres were determined to be approximately $70 \mathrm{~nm}$ after DLS measurements (Figure S2). The complexation was also confirmed with zeta potential measurements (Table S1), as the charge of the system decreased following the addition of the negatively charged AONs onto the PAs.

The biocompatilibity of nanofibrous and nanospherical systems was investigated by using the MCF-7 cell line with $24 \mathrm{~h}$ of culture (Figure S3). No significant decrease in cell viability was observed at $30 \mu \mathrm{M}$; accordingly, PA molecules were used at this concentration for in vitro experiments.

The cellular internalization of the PA/AON nanocomplexes was visualized by confocal microscopy following staining nuclei with TO-PRO-3 and actin filaments with phalloidin (red color shows FAM-AON). The PA/AON complexes were incubated for $4 \mathrm{~h}$ (Figure S4) and $24 \mathrm{~h}$ (Figure 3 ) to investigate their 


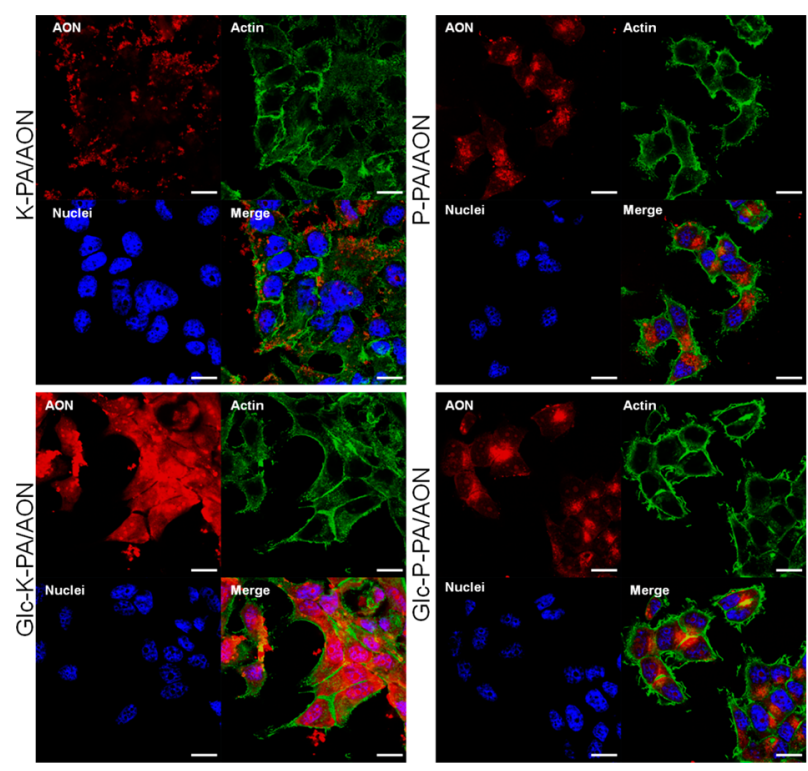

Figure 3. Twenty-four hours of cellular internalization of PA/AON complexes in MCF7 cells with FAM-AON in red, actin in green, and nuclei in blue. Scale bars: $20 \mu \mathrm{m}$.

cellular internalization. The nanofibers, and especially K-PA/ AON, were observed to bind better to the cell membrane, which suggests that the internalization of AON might be slower in nanofibers compared to nanospheres (Supporting Movies 1 and 2). During live imaging of cellular internalization, nanospheres were observed to be internalized during the first $5 \mathrm{~min}$, whereas cells only started to internalize nanofibers after $30 \mathrm{~min}$ of incubation. The rapid uptake of nanospheres could be due to their direct transduction through the cell membrane. The uptake after $24 \mathrm{~h}$ was also quantified with flow cytometry, and in contrast with our short-term internalization results, the cellular internalization of nanofibers was observed to be more efficient compared to that of nanospheres. These results show that, although nanofibers appear to enter the cells later than nanospheres (after $30 \mathrm{~min}$ ), they are able to deliver a greater amount of AONs into cells at $24 \mathrm{~h}$ compared to the nanospherical carrier system.

Because of the Warburg effect, glucose units can be used to increase the cellular uptake of nanostructures into cancer cells. This increase in uptake is a result of overexpressed glucose transporters on the cell surface. ${ }^{15}$ In accordance with this phenomenon, the cellular entry of Glc-K-PA/AON was found to be enhanced compared to that of K-PA/AON (Figure 4); however, no difference between the internalization of P-PA/ AON and Glc-P-PA/AON was observed. The reason behind this lack of enhancement in nanosphere carriers may be the fact that different internalization pathways are utilized for the entry of nanofibers and nanospheres. Consequently, internalization mechanisms of the PA delivery systems were assessed to determine whether this is the case.

Supramolecular shape of the nanostructures was shown to have a significant effect on the cellular internalization mechanism. ${ }^{40}$ Studies conducted with peptide-based materials revealed that cylindrical morphology results in both enhanced delivery efficiency and an increased number of potential binding sites compared to those of spherical counterparts due to the multivalent presentation of bioactive epitopes along the long-axis of the nanofiber. ${ }^{41-43}$ The possible mechanisms of internalization are direct penetration and endocytosis (clathrin-

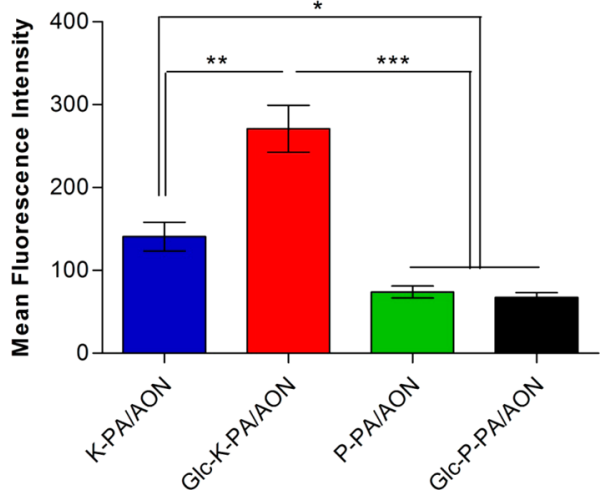

Figure 4. Cellular internalization of PA/AON complexes after $24 \mathrm{~h}$ quantified with flow cytometry. Error bars show SEM; two independent experiments were repeated with $n=3$ in each experiment. Student's $t$ test shows statistical significance with $* p<0.05$, $*^{*} p<$ 0.01 , and $* * * p<0.001$.

mediated endocytosis, caveolae-mediated endocytosis, and micropinocytosis), and the effect of various types of endocytosis inhibitors (amiloride, dynasore, chlorpromazine, and nystatin) on the internalization of nanofibers and nanospheres was determined through flow cytometry. Amiloride inhibits micropinocytosis, dynasore inhibits dynamin-dependent endocytosis, chlorpromazine inhibits clathrinmediated endocytosis, and nystatin inhibits caveolae-mediated endocytosis. ${ }^{44}$ Nearly no inhibition in uptake was observed for nanospheres (P-PA/AON and Glc-P-PA/AON) in the presence of endocytosis inhibitors, suggesting that nanosphere internalization occurs by direct transduction through the cell membrane (Figure 5). This result is consistent with our observations during live confocal imaging, where spheres entered into cells in the first $5 \mathrm{~min}$ of incubation. The smaller size of nanospheres might support their rapid entry into cells. ${ }^{45}$ There is a small decrease in the uptake of nanospheres in amiloride- and dynasore-treated cells, which suggested that spheres could enter cells via micropinocytosis and through dynamin-dependent pathways. This rapid and diffusionmediated internalization is a possible reason for the lack of additional uptake in glucose-conjugated nanospheres.

Nanofiber internalization, on the other hand, was inhibited by amiloride, dynasore, and chlorpromazine, suggesting that nanofiber uptake occurs by micropinocytosis, dynamin-dependent endocytosis, and clathrin-mediated endocytosis (Figure 5). Although Glc-K-PA/AON exhibited a decrease in uptake in response to amiloride treatment, no change was observed in $\mathrm{K}$ $\mathrm{PA} / \mathrm{AON}$, which might due to the effect of amiloride on the inhibition of the glucose transporter mechanism. ${ }^{46}$ These results show that the internalization of nanofibers is energydependent and that they are taken up by cells mostly by endocytosis, whereas nanospheres mostly enter cells passively. This difference in internalization pathway might result from the size and geometry of the self-assembled nanostructures, as membrane wrapping times of nanoparticles were previously shown to depend on their sizes and shapes. ${ }^{45}$ According to our results, nanospheres (which have smaller aspect ratios) can pass easily through the cell membrane, whereas high-aspect-ratio nanofibers require energy-dependent pathways for internalization. In addition, nanofiber internalization is slower and must be mediated through endocytic vesicles, whereas nanospheres are able to diffuse through the cell membrane following their internalization. Consequently, the overall internalization of the 


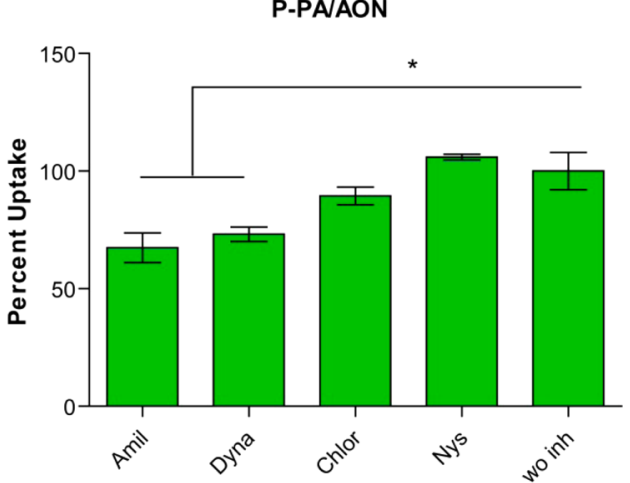

c)

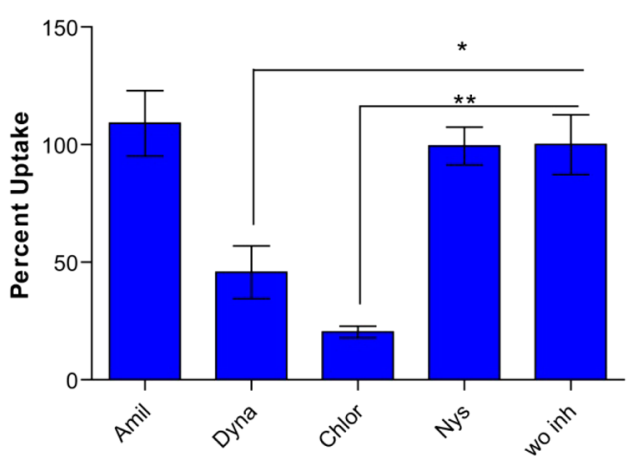

b)

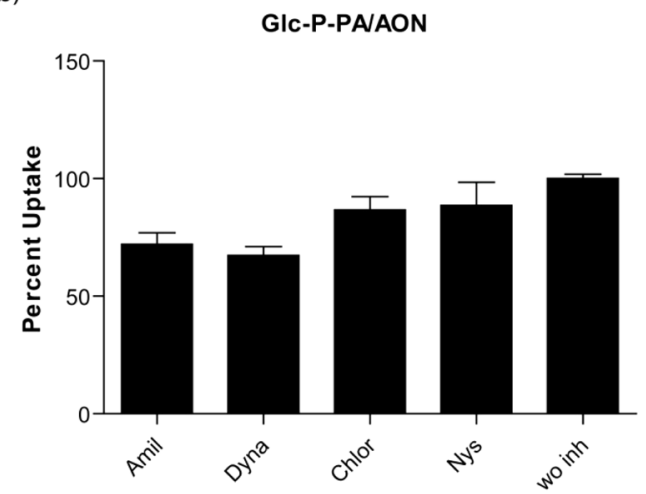

d)

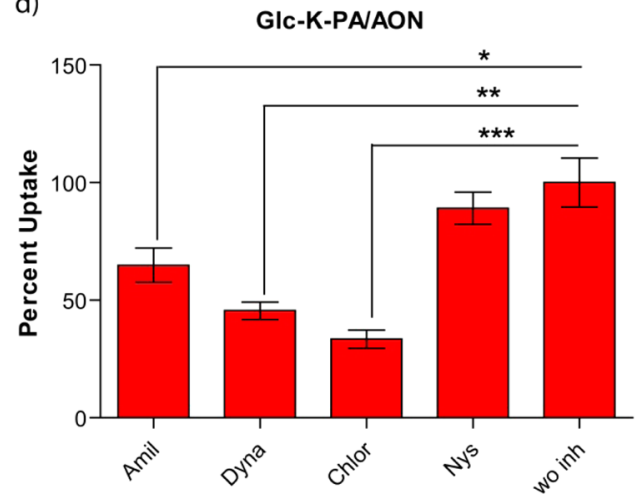

Figure 5. Uptake mechanism analyses of (a) P-PA/AON, (b) Glc-P-PA/AON, (c) K-PA/AON, and (d) Glc-K-PA/AON complexes. Percent uptake in MCF-7 cells after $24 \mathrm{~h}$ normalized to the without inhibitor group (wo inh). Amiloride (Amil), dynasore (Dyna), chlorpromazine (Chlor), and nystatin (Nys) were administered to cells to inhibit endocytosis pathways. Error bars show SEM; two independent experiments were repeated with $n$ $=3$ in each experiment. One-way ANOVA analysis was performed. Statistical significance is shown with $* p<0.05, * * p<0.01$, and $* * * p<0.001$.

nanofibers at $24 \mathrm{~h}$ was greater than that of nanospheres, as nanofibers cannot leave the intracellular environment whereas nanospheres can diffuse back out through the cell membrane.

Variances were also observed in the internalization mechanisms of glucose-conjugated PAs. In particular, the uptake of Glc-K-PA/AON was increased compared to that of $\mathrm{K}-\mathrm{PA} / \mathrm{AON}$ (Figure 3 and Figure 4) because the glucose moiety increases interactions between nanofibers and the cell membrane. The glucose transporter inhibitors phloridzin dihydrate (also known as phloridzin) and cytochalasin B were used to further analyze the effect of glucose conjugation on the internalization of nanofibrous AON delivery systems. The expression of glucose transporters GLUT1, GLUT2, GLUT5, ${ }^{47}$ GLUT12, ${ }^{23}$ and SGLT1 $^{48}$ was previously shown in MCF-7 cells. Of these, phloridzin specifically inhibits SGLT1 and SGLT2 and minimally inhibits GLUTs, ${ }^{49}$ and cytochalasin B inhibits glucose transport ${ }^{50}$ by inhibiting GLUT transporters (especially GLUT1-4). ${ }^{51}$ We observed a decrease in the internalization of Glc-K-PA/AON following phloridzin treatment, suggesting that the uptake of the glycopeptide nanofibrous oligonucleotide delivery system is mediated by its interactions with the sodium-dependent glucose transporter SGLT1 (Figure 6). Strikingly, phloridzin decreased the uptake of Glc-K-PA/AON almost to the level of K-PA/AON, demonstrating that glucose conjugation increases cellular internalization by promoting the binding of the nanofiber to glucose transporters. Even in the presence of GLUT and SGLT1 inhibitors, other glucose receptors might still be functional and may contribute to the internalization. ${ }^{23,52}$ On the other hand, it has been previously shown that the

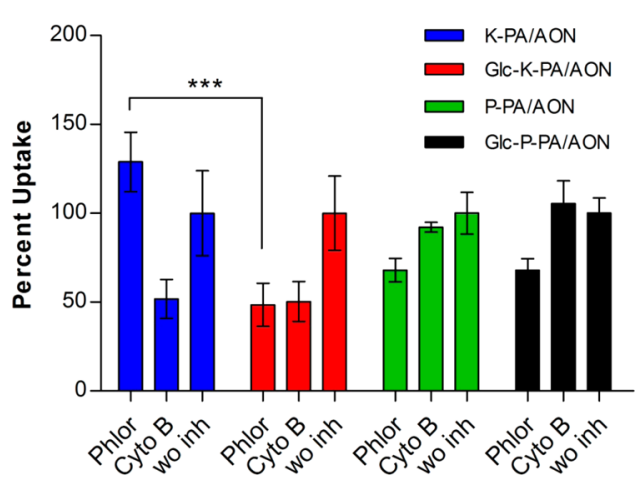

Figure 6. Glucose transporter-mediated uptake mechanisms of PA/ AON complexes. Percent uptake in MCF-7 cells after $24 \mathrm{~h}$ normalized to the without inhibitor group (wo inh). Phloridzin dihydrate (Phlor) and cytochalasin B (Cyto B) were administered to cells to inhibit glucose transporters. Error bars show SEM with $n=3$. Two-way ANOVA was performed. Statistical significance is shown with $* p<$ $0.05, * * p<0.01$, and $* * * p<0.001$.

internalization of glucose-conjugated nanoparticles may be facilitated by caveolae- and clathrin-mediated endocytosis. ${ }^{53-55}$ Although the chemical and biological inhibitors of specific cellular uptake mechanisms provide valuable tools for the study of cellular internalization mechanisms of nanomaterials, their interactions with the nanomaterials might change the overall characteristics of the nanomaterials, such as surface charge, which is an important determinant of cell uptake rate. 


\section{CONCLUSIONS}

In this study, we utilized self-assembled peptide amphiphile nanostructures with distinct geometries (nanofibers and nanospheres) to investigate the effect of the shape of the delivery system on the cellular internalization of oligonucleotides. We observed that the size and shape of the delivery system are important parameters for the cellular internalization of the ODN cargo. Whereas nanospheres were found to enter cells directly, nanofibers were internalized by dynamin- and clathrin-mediated endocytosis. The internalization of nanospheres was a rapid process, whereas the uptake of nanofibers took more time. However, fibers were found to carry more oligonucleotides into cells under long-term observation, as nanospheres are able to diffuse out of the cells despite their rapid uptake. Peptide amphiphiles were also functionalized with glucose units to produce glycopeptide delivery systems, which enhanced AON delivery through glucose transporter-mediated internalization in the nanofiber system (but did not yield any enhancement in performance in the nanosphere system due to differences in uptake mechanisms). Thus, glucose-functionalized, self-assembling peptide amphiphiles can be useful for clinical oligonucleotide delivery, although future carrier designs should also consider that the effectiveness of targeting and internalization-enhancing moieties may depend on the size and morphology of the carrier.

\section{EXPERIMENTAL SECTION}

Materials. 9-Fluorenylmethoxycarbonyl (Fmoc)- and tert-butoxycarbonyl (Boc)-protected amino acids, except glyco amino acid, [4$\left[\alpha-\left(2^{\prime}, 4^{\prime}\right.\right.$-dimethoxyphenyl) Fmoc-aminomethyl]phenoxy]acetamidonorleucyl-MBHA resin (Rink amide MBHA resin), and 2(1H-benzotriazol-1-yl)-1,1,3,3 tetramethyluronium hexafluorophosphate (HBTU), were purchased from NovaBiochem. Fmoc-Ser $[\beta$ $\mathrm{Glc}(\mathrm{OAc}) 4]-\mathrm{OH}$ was purchased from AAPPTec. Lauric acid and $N, N$ diisopropylethylamine (DIEA) were purchased from Merck. Other chemicals were purchased from Alfa Aesar or Sigma-Aldrich and used as provided. All water used was deionized water with a resistance of 18 $\mathrm{M} \Omega \mathrm{cm}$ (Millipore Milli-Q). Cell culture media, fetal bovine serum, penicillin-streptomycin, and trypsin were purchased from Gibco, Life Technologies. Bcl-2 antisense oligonucleotide (G-3129, Genasense)and fluorescein-6-amide (FAM)-labeled antisense oligonucleotide having the same sequence were donated by Genta Inc.

Peptide Synthesis and Characterization. 9-Fluorenylmethoxycarbonyl (Fmoc) chemistry was used to synthesize peptide amphiphiles and amphiphilic glycopeptides. PA molecules were constructed on MBHA rink amide resin (loading $=0.56 \mathrm{~mol} / \mathrm{g}$ ) and prepared on $0.25 \mathrm{mmol}$ scale. All amino acid couplings were manually performed with 2 equiv of Fmoc-protected amino acid, 1.95 equiv of HBTU, and 3 equiv of $N, N$-diisopropylethylamine (DIEA) in DMF for $3 \mathrm{~h}$. Lauric acid was conjugated to the $\mathrm{N}$-terminal of the peptide by the addition of the same reagents used for amino acid couplings. Each coupling reaction was monitored by ninhydrin test. Fmoc deprotections were performed with $20 \%$ piperidine/dimethylformamide (DMF) solution for $20 \mathrm{~min}$. Cleavage of the peptides from the resin was carried out with a mixture of trifluoroacetic acid (TFA)/ triisoproplysilane (TIS)/water at a ratio of 95:2.5:2.5 for $2 \mathrm{~h}$. Excess TFA was removed by rotary evaporation. The remaining viscous peptide solution was treated with ice-cold diethyl ether, and the resulting white pellet was freeze-dried. For glycopeptides, acetyl protecting groups on glucose were removed in solution phase with $\mathrm{NaOMe}$ (1.2 equiv excess with respect to acetyl groups, $2 \mathrm{M}$ ) in methanol. The reaction was carried out at room temperature for 3-4 h. For quenching the reaction, the solution was neutralized with a few drops of acetic acid. The solution was then collected, dissolved in water, and freeze-dried. All peptides were purified on an Agilent 1200 HPLC using an Agilent Zorbax 300SB-C8 $(21.2 \times 150 \mathrm{~mm})$ column with a water $(0.1 \%$ TFA $)$ /acetonitrile $(0.1 \%$ TFA $)$ gradient. The final purified peptides were characterized by reverse-phase HPLC on an Agilent 6530 accurate-Mass Q-TOF LC/MS equipped with an Agilent 1200 HPLC. A Phenomenex Luna $3 \mu$ C8 100A $(50 \times 3.00 \mathrm{~mm})$ column and a water $(0.1 \%$ formic acid $)$ /acetonitrile ( $0.1 \%$ formic acid) gradient were used as stationary phase and mobile phase, respectively. Peptides with a purity of more than $95 \%$ were used in the experiments.

Circular Dichroism. PA samples were prepared at a final concentration of $200 \mu \mathrm{M}$ in water, and $\mathrm{PA} / \mathrm{AON}$ combinations were prepared at a 30:1 PA/AON molar ratio. Quartz cuvettes with $1 \mathrm{~mm}$ path lengths were used for all measurements. Circular dichroism analysis was performed with a J-815 Jasco spectrophotometer in the far UV region. Parameters were selected as follows: integration time of 4 $\mathrm{s}$, bandwidth of $1 \mathrm{~nm}$, and data pitch of $0.1 \mathrm{~nm}$. An average of three readings between 190 and $260 \mathrm{~nm}$ were collected for each sample. Ellipticity was converted to molar ellipticity with the unit degree $\mathrm{cm}^{2}$ $\mathrm{dmol}^{-1}$.

Transmission Electron Microscopy. TEM samples were prepared on a 200-mesh copper grid. Dilute solutions of PA/AON complexes at $0.05 \%(\mathrm{w} / \mathrm{v})$ and $50 \mathrm{ng} / \mu \mathrm{L}$ of $\mathrm{AON}$ concentrations were used for TEM analysis. Samples were dropped on copper grids, incubated for $5 \mathrm{~min}$, and stained with $2 \mathrm{wt} \%$ uranyl acetate for $3 \mathrm{~min}$ following the removal of excess solution. After washing, samples were air-dried, and images were obtained with a FEI Tecnai G2 F30 TEM at $200 \mathrm{keV}$.

Maintenance of Cells and Transfection with PA and PA/AON Complexes. Cells were maintained in standard medium containing Dulbecco's modified eagle medium (DMEM) with $10 \%$ fetal bovine serum (FBS) and $1 \%$ penicillin-streptomycin and passaged at cell confluency between 80 and $90 \%$ using trypsin-EDTA. In transfection experiments, $\mathrm{PA} / \mathrm{AON}$ complexes were prepared in $\mathrm{ddH}_{2} \mathrm{O}$ to have a molar ratio of $30: 1$ and administered in serum-free medium ( $1 \%$ penicillin-streptomycin-containing DMEM).

Cellular Internalization of PA/AON Complexes. Thirteen millimeter glass coverslips were placed in 24-well plates, and $4 \times$ $10^{4}$ MCF7 cells per well were seeded in standard medium. After $24 \mathrm{~h}$, medium was discarded, and PA/AON complexes were administered in serum-free medium. The AON final concentration was set to $1 \mu \mathrm{M}$, and the PA concentration was adjusted accordingly. After incubation, cells were washed with PBS three times, fixed with $4 \%$ paraformaldehyde, and stained with phalloidin and TO-PRO-3. Cells were visualized with a laser scanning confocal microscope (LSM 510, Zeiss).

Mechanisms of Internalization. Small molecule chemical inhibitors were used to inhibit specific internalization pathways for investigating the internalization mechanism of PA/AON complexes. Inhibitor concentrations were adjusted in a preliminary experiment, and the maximum dose that did not induce cell death was selected for testing. Thus, $8 \times 10^{4}$ MCF-7 cells/well were seeded in standard medium in 24-well plates. After $24 \mathrm{~h}$, medium was removed, and 400 $\mu \mathrm{L}$ of serum-free medium was added. Then, $50 \mu \mathrm{L}$ inhibitor solutions were administered to a final concentration of $100 \mu \mathrm{M}$ amiloride, 20 $\mu \mathrm{M}$ dynasore, $0.2 \mu \mathrm{g} / \mathrm{mL}$ of chlorpromazine, or $10 \mu \mathrm{g} / \mathrm{mL}$ of nystatin. In addition, final concentrations of $1000 \mu \mathrm{M}$ phloridzin dihydrate and $50 \mu \mathrm{M}$ cytochalasin $\mathrm{B}$ were used in glucose transporter inhibition studies. After $1 \mathrm{~h}$ of incubation, $\mathrm{PA} / \mathrm{AON}$ complexes were administered to have a final concentration of $30 \mu \mathrm{M}$ PA and $1 \mu \mathrm{M}$ AON. Cells were incubated for another $24 \mathrm{~h}$ and collected in eppendorf tubes following trypsinization. After washing with PBS two times and centrifugation at $1500 \mathrm{rpm}$ for $5 \mathrm{~min}$, cells were dissolved in PBS. Cells were then trypsinized, kept on ice, and analyzed with a Guava easycyte flow cytometer (Milipore). Cells were gated by side scatter channel (SSC) and forward scatter channel (FSC) using a nontreated control. The fluorescence intensity of FAM-AONs was measured with the green channel. 


\section{ASSOCIATED CONTENT}

\section{S Supporting Information}

The Supporting Information is available free of charge on the ACS Publications website at DOI: 10.1021/acsami.6b01526.

Liquid chromatogram and mass spectra of PAs, size distribution of $\mathrm{PA} / \mathrm{AON}$ nanospheres, cytotoxicity of PAs administered to MCF7 cells for $24 \mathrm{~h}, 4 \mathrm{~h}$ internalization of $\mathrm{PA} / \mathrm{AON}$ complexes in MCF-7 cells, and zeta potentials of $\mathrm{PA}$ and $\mathrm{PA} / \mathrm{AON}$ complexes (PDF)

Live confocal imaging of cells treated with lysotracker and K-PA/AON complex for $6 \mathrm{~h}$ (AVI)

Live confocal imaging of cells treated with lysotracker and P-PA/AON complex for $6 \mathrm{~h}$ (AVI)

\section{AUTHOR INFORMATION}

\section{Corresponding Authors}

*E-mail: atekinay@unam.bilkent.edu.tr.

*E-mail: moguler@unam.bilkent.edu.tr.

\section{Author Contributions}

${ }^{\#}$ D.M. and M.S.E. contributed equally to this work.

Notes

The authors declare no competing financial interest.

\section{ACKNOWLEDGMENTS}

We would like to thank M. Guler for TEM imaging, and G. Cinar and A.D. Ozkan for fruitful scientific discussion. D.M. and M.S.E are supported by TUBITAK-BIDEB fellowships. This work is partly supported by TUBITAK $114 Z 562$ and $113 \mathrm{~T} 045$

\section{REFERENCES}

(1) Kurreck, J. Therapeutic Oligonucleotides. RSC Biomol. Sci. 2008, $0,1-102$.

(2) Bouchard, P.; Hutabarat, R.; Thompson, K. Discovery and Development of Therapeutic Aptamers. Annu. Rev. Pharmacol. Toxicol. 2010, 50, 237-257.

(3) Kole, R.; Krainer, A. R.; Altman, S. RNA therapeutics: beyond RNA interference and antisense oligonucleotides. Nat. Rev. Drug Discovery 2012, 11 (2), 125-140.

(4) Bennett, C. F.; Swayze, E. E. RNA Targeting Therapeutics: Molecular Mechanisms of Antisense Oligonucleotides as a Therapeutic Platform. Annu. Rev. Pharmacol. Toxicol. 2010, 50, 259-293.

(5) Patil, S. D.; Rhodes, D. G.; Burgess, D. J. DNA-Based Therapeutics and DNA Delivery Systems: A Comprehensive Review. AAPS J. 2005, 7 (1), E61-E77.

(6) Juliano, R.; Bauman, J.; Kang, H.; Ming, X. Biological Barriers to Therapy with Antisense and siRNA Oligonucleotides. Mol. Pharmaceutics 2009, 6 (3), 686-695.

(7) Huang, L.; Liu, Y. In Vivo Delivery of RNAi with Lipid-Based Nanoparticles. Annu. Rev. Biomed. Eng. 2011, 13, 507-530.

(8) Ewert, K. K.; Zidovska, A.; Ahmad, A.; Bouxsein, N. F.; Evans, H. M.; McAllister, C. S.; Samuel, C. E.; Safinya, C. R. Cationic LiposomeNucleic Acid Complexes for Gene Delivery and Silencing: Pathways and Mechanisms for Plasmid DNA and siRNA. Top. Curr. Chem. 2010, 296, 191-226.

(9) Zhu, L.; Mahato, R. I. Lipid and Polymeric Carrier-Mediated Nucleic Acid Delivery. Expert Opin. Drug Delivery 2010, 7 (10), 12091226.

(10) Rosi, N. L.; Giljohann, D. A.; Thaxton, C. S.; Lytton-Jean, A. K. R.; Han, M. S.; Mirkin, C. A. Oligonucleotide-Modified Gold Nanoparticles for Intracellular Gene Regulation. Science 2006, 312 (5776), 1027-1030.
(11) McBain, S. C.; Yiu, H. H. P.; Dobson, J. Magnetic Nanoparticles for Gene and Drug Delivery. Int. J. Nanomed. 2008, 3 (2), 169-180. (12) Zhu, S. G.; Xiang, J. J.; Li, X. L.; Shen, S. R.; Lu, H. B.; Zhou, J.; Xiong, W.; Zhang, B. C.; Nie, X. M.; Zhou, M.; Tang, K.; Li, G. Y. Poly(L-lysine)-Modified Silica Nanoparticles for the Delivery of Antisense Oligonucleotides. Biotechnol. Appl. Biochem. 2004, 39, 179-187.

(13) Simone, E. A.; Dziubla, T. D.; Muzykantov, V. R. Polymeric Carriers: Role of Geometry in Drug Delivery. Expert Opin. Drug Delivery 2008, 5 (12), 1283-1300.

(14) Shang, L.; Nienhaus, K.; Nienhaus, G. U. Engineered Nanoparticles Interacting with Cells: Size Matters. J. Nanobiotechnol. 2014, 12, 5 .

(15) Macheda, M. L.; Rogers, S.; Best, J. D. Molecular and Cellular Regulation of Glucose Transporter (GLUT) Proteins in Cancer. J. Cell. Physiol. 2005, 202 (3), 654-662.

(16) Vander Heiden, M. G.; Cantley, L. C.; Thompson, C. B. Understanding the Warburg Effect: The Metabolic Requirements of Cell Proliferation. Science 2009, 324 (5930), 1029-1033.

(17) Warburg, O. On the Origin of Cancer Cells. Science 1956, 123 (3191), 309-314.

(18) Hanahan, D.; Weinberg, R. A. Hallmarks of Cancer: The Next Generation. Cell 2011, 144 (5), 646-674.

(19) Krzeslak, A.; Wojcik-Krowiranda, K.; Forma, E.; Jozwiak, P.; Romanowicz, H.; Bienkiewicz, A.; Brys, M. Expression of GLUT1 and GLUT3 Glucose Transporters in Endometrial and Breast Cancers. Pathol. Oncol. Res. 2012, 18 (3), 721-728.

(20) Chan, D. A.; Sutphin, P. D.; Nguyen, P.; Turcotte, S.; Lai, E. W.; Banh, A.; Reynolds, G. E.; Chi, J. T.; Wu, J.; Solow-Cordero, D. E.; Bonnet, M.; Flanagan, J. U.; Bouley, D. M.; Graves, E. E.; Denny, W. A.; Hay, M. P.; Giaccia, A. J. Targeting GLUT1 and the Warburg Effect in Renal Cell Carcinoma by Chemical Synthetic Lethality. Sci. Transl. Med. 2011, 3 (94), 94ra70.

(21) Yun, J.; Rago, C.; Cheong, I.; Pagliarini, R.; Angenendt, P.; Rajagopalan, H.; Schmidt, K.; Willson, J. K.; Markowitz, S.; Zhou, S.; Diaz, L. A., Jr.; Velculescu, V. E.; Lengauer, C.; Kinzler, K. W.; Vogelstein, B.; Papadopoulos, N. Glucose Deprivation Contributes to The Development of KRAS Pathway Mutations in Tumor Cells. Science 2009, 325 (5947), 1555-1559.

(22) Grover-McKay, M.; Walsh, S. A.; Seftor, E. A.; Thomas, P. A.; Hendrix, M. J. Role for Glucose Transporter 1 Protein in Human Breast Cancer. Pathol. Oncol. Res. 1998, 4 (2), 115-120.

(23) Calvo, M. B.; Figueroa, A.; Pulido, E. G.; Campelo, R. G.; Aparicio, L. A. Potential Role of Sugar Transporters in Cancer and Their Relationship with Anticancer Therapy. Int. J. Endocrinol. 2010, 2010, 1.

(24) Casneuf, V. F.; Fonteyne, P.; Van Damme, N.; Demetter, P.; Pauwels, P.; de Hemptinne, B.; De Vos, M.; Van de Wiele, C.; Peeters, M. Expression of SGLT1, Bcl-2 and p53 in Primary Pancreatic Cancer Related to Survival. Cancer Invest. 2008, 26 (8), 852-859.

(25) Ishikawa, N.; Oguri, T.; Isobe, T.; Fujitaka, K.; Kohno, N. SGLT Gene Expression in Primary Lung Cancers and Their Metastatic Lesions. Jpn. J. Cancer Res. 2001, 92 (8), 874-879.

(26) Briasoulis, E.; Judson, I.; Pavlidis, N.; Beale, P.; Wanders, J.; Groot, Y.; Veerman, G.; Schuessler, M.; Niebch, G.; Siamopoulos, K.; Tzamakou, E.; Rammou, D.; Wolf, L.; Walker, R.; Hanauske, A. Phase I Trial of 6-h Infusion of Glufosfamide, a New Alkylating Agent with Potentially Enhanced Selectivity for Tumors that Overexpress Transmembrane Glucose Transporters: A Study of the European Organization for Research and Treatment of Cancer Early Clinical Studies Group. J. Clin. Oncol. 2000, 18 (20), 3535-3544.

(27) Fu, Y.; Li, S.; Zu, Y.; Yang, G.; Yang, Z.; Luo, M.; Jiang, S.; Wink, M.; Efferth, T. Medicinal Chemistry of Paclitaxel and Its Analogues. Curr. Med. Chem. 2009, 16 (30), 3966-3985.

(28) Cao, J.; Cui, S.; Li, S.; Du, C.; Tian, J.; Wan, S.; Qian, Z.; Gu, Y.; Chen, W. R.; Wang, G. Targeted Cancer Therapy with a 2deoxyglucose-Based Adriamycin Complex. Cancer Res. 2013, 73 (4), $1362-1373$ 
(29) Amanlou, M.; Heidari, Z.; Siadat, S. D.; Aghasadeghi, M. R.; Ghorbani, M.; Ebrahimi, S. E.; Sadat, S. M.; Hajmohammadi, M.; Arabzadeh, A. J.; Hekmat, S.; Alaei-Beirami, M.; Saraji, A. A.; Moghaddam, H. F.; Alavidjeh, M. S.; Delbaz, S. A.; DashtbaniRoozbehani, A.; Ardestani, M. S. Nanosized Tamoxifen-PorphyrinGlucose [TPG] Conjugate: Novel Selective Anti-Breast-Cancer Agent, Synthesis and In Vitro Evaluations. Med. Chem. 2013, 9 (4), 526-538.

(30) Jain, K.; Kesharwani, P.; Gupta, U.; Jain, N. K. A Review of Glycosylated Carriers for Drug Delivery. Biomaterials 2012, 33 (16), 4166-4186.

(31) Calvaresi, E. C.; Hergenrother, P. J. Glucose Conjugation for the Specific Targeting and Treatment of Cancer. Chem. Sci. 2013, 4 (6), 2319-2333.

(32) Wang, J.; Yin, C.; Tang, G.; Lin, X.; Wu, Q. GlucoseFunctionalized Multidrug-Conjugating Nanoparticles Based on Amphiphilic Terpolymer with Enhanced Anti-Tumorous Cell Cytotoxicity. Int. J. Pharm. 2013, 441 (1-2), 291-298.

(33) Yin, L. G.; Dalsin, M. C.; Sizovs, A.; Reineke, T. M.; Hillmyer, M. A. Glucose-Functionalized, Serum-Stable Polymeric Micelles from the Combination of Anionic and RAFT Polymerizations. Macromolecules 2012, 45 (10), 4322-4332.

(34) Bulut, S.; Erkal, T. S.; Toksoz, S.; Tekinay, A. B.; Tekinay, T.; Guler, M. O. Slow Release and Delivery of Antisense Oligonucleotide Drug by Self-Assembled Peptide Amphiphile Nanofibers. Biomacromolecules 2011, 12 (8), 3007-3014.

(35) Guler, M. O.; Claussen, R. C.; Stupp, S. I. Encapsulation of Pyrene within Self-Assembled Peptide Amphiphile Nanofibers. J. Mater. Chem. 2005, 15 (42), 4507-4512.

(36) Mumcuoglu, D.; Sardan, M.; Tekinay, T.; Guler, M. O.; Tekinay, A. B. Oligonucleotide Delivery with Cell Surface Binding and Cell Penetrating Peptide Amphiphile Nanospheres. Mol. Pharmaceutics 2015, 12 (5), 1584-1591.

(37) Whitmore, L.; Wallace, B. A. Protein Secondary Structure Analyses from Circular Dichroism Spectroscopy: Methods and Reference Databases. Biopolymers 2008, 89 (5), 392-400.

(38) Sadler, K.; Eom, K. D.; Yang, J. L.; Dimitrova, Y.; Tam, J. P. Translocating Proline-Rich Peptides from the Antimicrobial Peptide Bactenecin 7. Biochemistry 2002, 41 (48), 14150-14157.

(39) Yoon, Y.-R.; Lim, Y.-b.; Lee, E.; Lee, M. Self-Assembly of a Peptide Rod-Coil: A Polyproline Rod and a Cell-Penetrating Peptide Tat Coil. Chem. Commun. 2008, 16, 1892-1894.

(40) Hu, X.; Hu, J.; Tian, J.; Ge, Z.; Zhang, G.; Luo, K.; Liu, S. Polyprodrug Amphiphiles: Hierarchical Assemblies for Shape-Regulated Cellular Internalization, Trafficking, and Drug Delivery. J. Am. Chem. Soc. 2013, 135 (46), 17617-17629.

(41) Lim, Y. b.; Lee, E.; Lee, M. Controlled Bioactive Nanostructures from Self-Assembly of Peptide Building Blocks. Angew. Chem., Int. Ed. 2007, 46 (47), 9011-9014.

(42) Moyer, T. J.; Kassam, H. A.; Bahnson, E. S.; Morgan, C. E.; Tantakitti, F.; Chew, T. L.; Kibbe, M. R.; Stupp, S. I. Shape-Dependent Targeting of Injured Blood Vessels by Peptide Amphiphile Supramolecular Nanostructures. Small 2015, 11 (23), 2750-2755.

(43) Mammadov, R.; Cinar, G.; Gunduz, N.; Goktas, M.; Kayhan, H.; Tohumeken, S.; Topal, A. E.; Orujalipoor, I.; Delibasi, T.; Dana, A. Virus-Like Nanostructures for Tuning Immune Response. Sci. Rep. 2015, 5, 16728.

(44) Iversen, T. G.; Skotland, T.; Sandvig, K. Endocytosis and Intracellular Transport of Nanoparticles: Present Knowledge and Need for Future Studies. Nano Today 2011, 6 (2), 176-185.

(45) Chithrani, B. D.; Chan, W. C. W. Elucidating the Mechanism of Cellular Uptake and Removal of Protein-Coated Gold Nanoparticles of Different Sizes and Shapes. Nano Lett. 2007, 7 (6), 1542-1550.

(46) Goto, Y.; Kida, K.; Kaino, Y.; Ito, T.; Matsuda, H. Inhibitory Effect of Amiloride on Glucose Transport in Isolated Rat Adipocytes. Diabetes Res. Clin. Pract. 1993, 20 (1), 1-5.

(47) ZamoraLeon, S. P.; Golde, D. W.; Concha, I. I.; Rivas, C. I.; DelgadoLopez, F.; Baselga, J.; Nualart, F.; Vera, J. C. Expression of the Fructose Transporter GLUT5 in Human Breast Cancer. Proc. Natl. Acad. Sci. U. S. A. 1996, 93 (5), 1847-1852.
(48) Janku, F.; Huang, H. J.; Angelo, L. S.; Kurzrock, R. A KinaseIndependent Biological Activity for Insulin Growth Factor-1 Receptor (IGF-1R): Implications for Inhibition of the IGF-1R Signal. Oncotarget 2013, 4 (3), 463-473.

(49) Ehrenkranz, J. R. L.; Lewis, N. G.; Kahn, C. R.; Roth, J. Phlorizin: A Review. Diabetes/Metab. Res. Rev. 2005, 21 (1), 31-38.

(50) Estensen, R. D.; Plagemann, P. G. Cytochalasin B: Inhibition of Glucose and Glucosamine Transport. Proc. Natl. Acad. Sci. U. S. A. 1972, 69 (6), 1430-1434.

(51) Augustin, R. The Protein Family of Glucose Transport Facilitators: It's Not Only About Glucose After All. IUBMB Life 2010, 62 (5), 315-333.

(52) Rogers, S.; Macheda, M. L.; Docherty, S. E.; Carty, M. D.; Henderson, M. A.; Soeller, W. C.; Gibbs, E. M.; James, D. E.; Best, J. D. Identification of a Novel Glucose Transporter-Like Protein-GLUT12. Am. J. Physiol. Endocrinol. Metab. 2002, 282 (3), E733-E738.

(53) Moros, M.; Hernáez, B.; Garet, E.; Dias, J. T.; Sáez, B.; Grazú, V.; González-Fernández, Á.; Alonso, C.; de la Fuente, J. s. M. Monosaccharides versus PEG-Functionalized NPs: Influence in the Cellular Uptake. ACS Nano 2012, 6 (2), 1565-1577.

(54) Jiang, X.; Xin, H.; Ren, Q.; Gu, J.; Zhu, L.; Du, F.; Feng, C.; Xie, Y.; Sha, X.; Fang, X. Nanoparticles of 2-Deoxy-D-Glucose Functionalized Poly (ethylene glycol)-co-poly (trimethylene carbonate) for Dual-Targeted Drug Delivery in Glioma Treatment. Biomaterials 2014, 35 (1), 518-529.

(55) Wang, Z.; Tiruppathi, C.; Minshall, R. D.; Malik, A. B. Size and Dynamics of Caveolae Studied Using Nanoparticles in Living Endothelial Cells. ACS Nano 2009, 3 (12), 4110-4116. 